\title{
Photonic Crystal Fiber Fabry-Perot Interferometers With High-Reflectance Internal Mirrors
}

\author{
Rong $\mathrm{FAN}^{1,2,3}$, Yuanbin $\mathrm{HOU}^{1 *}$, and Wei $\mathrm{SUN}^{2,3}$ \\ ${ }^{1}$ School of Electric and Control Engineering, Xi'an University of Science and Technology, Xi'an, 710054, China \\ ${ }^{2}$ Chongqing Research Institute of China Coal Technology Engineering Group, Chongqing, 400039, China \\ ${ }^{3}$ Chongqing Engineering Research Center of Key Technologies of Mine Internet of Things, Chongqing, 400039, China \\ ${ }^{*}$ Corresponding author: Yuanbin HOU E-mail: houyb@xust.edu.cn
}

\begin{abstract}
We demonstrated an in-line micro fiber-optic Fabry-Perot interferometer with an air cavity which was created by multi-step fusion splicing a muti-mode photonic crystal fiber (MPCF) to a standard single mode fiber (SMF). The fringe visibility of the interference pattern was up to $20 \mathrm{~dB}$ by reshaping the air cavity. Experimental results showed that such a device could be used as a highly sensitive strain sensor with the sensitivity of $4.5 \mathrm{pm} / \mu \varepsilon$. Moreover, it offered some other outstanding advantages, such as the extremely compact structure, easy fabrication, low cost, and high accuracy.
\end{abstract}

Keywords: Fiber-optic sensors, Fabry-Perot interferometer, photonic crystal fiber, strain measurement

Citation: Rong FAN, Yuanbin HOU, and Wei SUN, "Photonic Crystal Fiber Fabry-Perot Interferometers With High-Reflectance Internal Mirrors," Photonic Sensors, 2015, 5(2): 97-101.

\section{Introduction}

All fiber in-line Fabry-Perot interferometers (FPIs) have advantages of the compact size, high resolution, excellent stability, and insensitivity to temperatures $[1,2]$. So researchers have been developing different methods to fabricate the air cavity based on different techniques, for example wet chemical etching [3, 4], laser micromachining $[5,6]$, and splicing different types of optical fibers with the commercialized fusion splicer [7-12]. Most of the above-mentioned FPIs can be good choices for strain sensing since a minute change in the cavity size leads to a detectable shift in the interference pattern $[4-6,8,9,11,12]$. However, some of these fabrication methods are difficult to implement since they require a complex manufacturing process or expensive equipment. Hence, for its simplicity, the fabrication method based on splicing two different optical fibers is an attractive option. This method has some inherent challenges, for example it is necessary to create a fusion arc splicing program and design an overall process to guarantee perfect reflective mirrors. The highest fringe visibility of the FPI is about $38 \mathrm{~dB}$, which is formed by pressuring a photonic crystal fiber with a conventional single mode fiber during fusion splicing [12]. So the complicated air inflation equipment is essential in the fabrication process.

We demonstrated a high-quality micro FPI which was fabricated by multi-step fusion splicing a multi-mode photonic crystal fiber (MPCF) to a standard single mode fiber (SMF). The micro-FPIs were entirely embedded in the fiber and exhibited excellent fringe visibility of up to $20 \mathrm{~dB}$ due to the mirror-finish quality of two cavity surfaces, which 
was superior to that of most FPIs based on the fusion splicing method [7-11]. The strain sensitivity of the device can be enhanced to $4.5 \mathrm{pm} / \mu \varepsilon$ by reshaping the air cavity created in the fiber. So this kind of FPI has the features of the extremely compact structure, easy fabrication, low cost, and high accuracy.

\section{Sensor fabrication and operation principle}

The cross section of the utilized MPCF is shown in Fig. 1. It had the core and cladding diameters of about $5 \mu \mathrm{m}$ and $114 \mu \mathrm{m}$, respectively. The diameter of the cladding holes in the MPCF was about $11 \mu \mathrm{m}$. A conventional arc fusion splicing machine (FurukawaS176) was used. Note that the manual operation mode of the fusion splicer was used to fabricate the in-line air cavity sample.
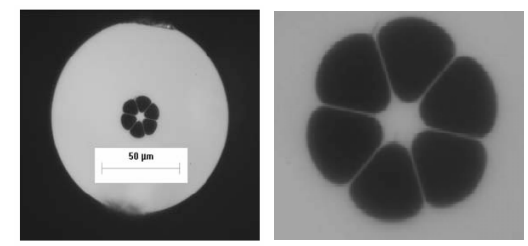

Fig. 1 Cross section of the MPCF.

We developed an optimized multi-step procedure by attempting several experiments with a wide range of splice parameters. The resulting parameters were as follows: arc power of 170 units, pre-fuse time of $100 \mathrm{~ms}$, arc duration of $700 \mathrm{~ms}$, and $z$-push distance of $5 \mu \mathrm{m}$. The cleaved ends of the SMF and MPCF were placed in the left and right fiber holders, respectively. Under these splicing conditions, the temperature achieved during the arc discharge would be high enough to exceed the MPCF softening point, and therefore the surface tension would overcome the viscosity. This not only formed a joint, but also collapsed the fine voids of the MPCF [13]. As a result, a part of air originally inside the voids could be trapped, and the rapidly expanding gases could induce a micro-bubble at the SMF-MPCF interface, as shown in Fig. 2(a). It could be seen that there was a quasi-spherical air cavity embedded in the fiber, whose interference pattern was observed by use of a 3-dB coupler, a broadband light source, and an optical spectrum analyzer (OSA, Si720, Micron Optics, USA), as shown in Fig. 2(b). The device exhibited a true and stable sinusoidal interference fringe with the visibility of about $7 \mathrm{~dB}$, which has been used to measure strain at high temperatures [9]. However, the optical performance and the sensitivity of the device strongly depended on the shape of the air cavity embedded in the fiber [12], so another two arc discharges were applied to change the shape of the air cavity as the fiber was not moved in the holders. Figure 2(c) illustrates the photo of the device after the second arc discharge. It could be seen that the shape of the bubble was still a quasi-spherical but the polar and equator diameters simultaneously increased due to the thermal expansion of air trapped in the cavity. The fringe visibility of the interference pattern increased to about $10 \mathrm{~dB}$, as shown in Fig. 2(d). We continuously applied the third arc discharge to the fiber, and the spherical air bubble was reshaped into an elliptical one, as shown in Fig.2(e). The polar diameter of the air cavity was greater than those of SMF and MPCF, respectively. Moreover, the MPCF has been tapered due to the complete collapse of air holes in the cladding as enough arc discharge was applied to the fiber. The corresponding interference pattern of the device is shown in Fig.2(f). We could see that the fringe visibility was up to $20 \mathrm{~dB}$, which was about three times of that of the FPI in [9]. The free spectral range (FSR) of the interference pattern is given by $F S R=\lambda^{2} / 2 n L$, where $\lambda$ is the wavelength of light, $n$ is the refractive index of the medium, i.e. air, trapped inside the bubble, $L$ is the cavity length of the air bubble created in the spliced joint. Using the measured value of $F S R=12.65 \mathrm{~nm}$ and $\lambda=1545 \mathrm{~nm}$, we calculated the cavity length of the air cavity as $94 \mu \mathrm{m}$, which agreed with the measured result of about $91 \mu \mathrm{m}$. In addition, we dipped the taper with different $\mathrm{NaCl}$ solutions and found that the reflected spectrum remained unchanged. So the taper had no effect on the interference pattern. As we continuously applied the forth arc discharge to the 
device, both of the air cavity and MPCF would crack. Therefore, in this case, three arc discharges were the best choice, and over $80 \%$ of the samples were formed if the fibers were properly cleaved.
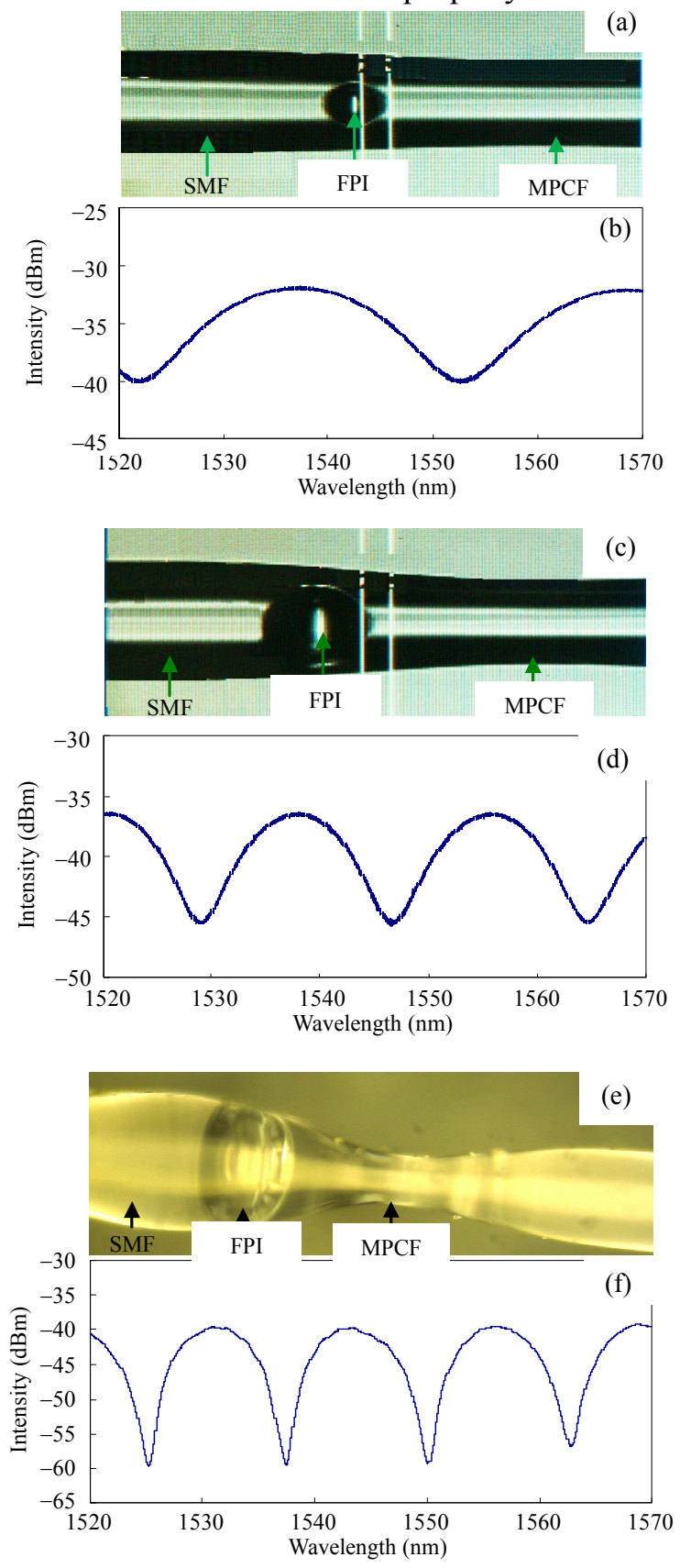

Fig. 2 Fabricated FPIs and their corresponding interference spectra under different arc discharges: (a) microscope image of the created air bubble after the first arc discharge, (b) the reflected spectrum of the FPI after the first arc discharge, (c) microscope image of the created air bubble after the second arc discharge, (d) the reflected spectrum of the FPI after the second arc discharge, (e) microscope image of the created air bubble after the third arc discharge, and (f) the reflected spectrum of the FPI after the third arc discharge.

\section{Experimental results and discussion}

To investigate the responses of the air-cavitybased FPI samples to the applied tensile strain, a pair of translation stages with the resolution of $10 \mu \mathrm{m}$ was used. The FPI with a cavity length of about $91 \mu \mathrm{m}$, as shown in Fig. 2(e), was fixed between two translation stages separated by a distance of $1 \mathrm{~m}$. The reflected optical spectra obtained were functions of the imposed tensile strain ranging from $0 \mu \varepsilon$ to $1570 \mu \varepsilon$, as shown in Fig. 3. We can see that the wavelength shows a red shift with an increment of the strain, which results from the fact that a longitudinal strain applied to the FPI optical cavity will change the cavity physical length and therefore change the FPI cavity reflection transfer function. As the phase shift is less than $2 \pi$, the wavelength shift of the interference spectrum as a function of the tensile strain applied to the air-cavity based FPI can be expressed by $\Delta \lambda_{m}=\varepsilon_{z} \lambda$. This indicates that the applied strain is directly proportional to the spectral shift of the interference fringe. So the characteristics spectral positions such as the interference peak, the central point of the interferogam or the interference valley can be monitored to measure the external strain. In our experiment, the wavelength shift of the interference fringe around $1537.5 \mathrm{~nm}$ was measured while the tensile strain increased from $0 \mu \varepsilon$ to $1570 \mu \varepsilon$, which is shown in Fig. 4. It was found that the wavelength shift of the FPI had a linear relationship with the applied strain without hysteresis. The strain sensitivity of $4.5 \mathrm{pm} / \mu \varepsilon$ could be obtained by linear fitting the curve, which was about twice of that FPI in [9]. This is mainly due to the fact that the micro air cavity exhibits an ellipsoid shape, and its strain sensitivity is dependent on the cavity volume [12]. Since the sensors cross thermo response is relevant for strain measurement applications, the thermal sensitivity for such a sensor was investigated with a high-temperature oven (Lenton 1200) for a temperature range between 0 and $500{ }^{\circ} \mathrm{C}$ with a step 
of $50{ }^{\circ} \mathrm{C}$. Experimental results showed that the interference spectrum hardly shifted in the whole process, that is to say, the interference fringe was insensitive to temperature, which was the same as that in [9]. Therefore, such a sensor can also be used to accurately measure the strain at high temperatures, and what's the most important is that the temperature compensation may not be required for most of strain sensing applications.

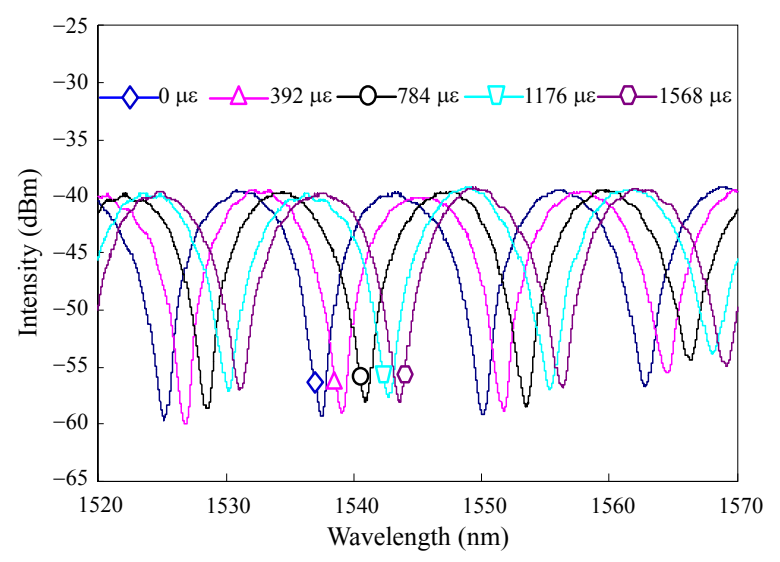

Fig. 3 Reflected spectra of the air-cavity-based FPI sample as the tensile strain increases from $0 \mu \varepsilon$ to $1570 \mu \varepsilon$.

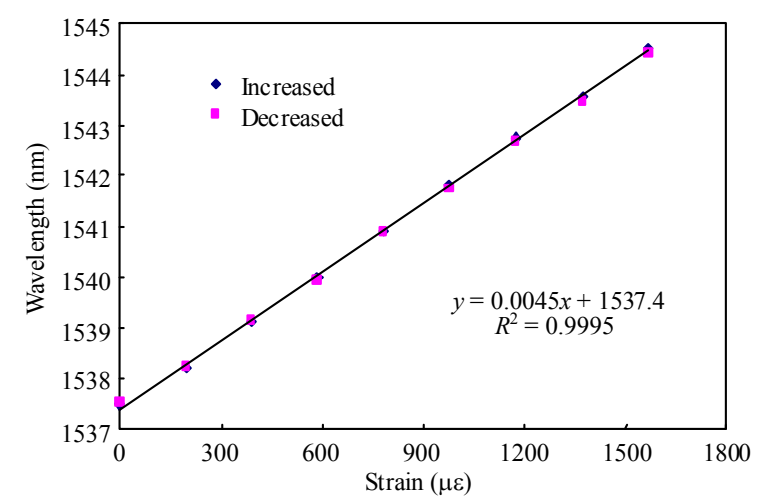

Fig. 4 Wavelength shift of the interference spectrum around $1537 \mathrm{~nm}$ as a function of the tensile strain applied to the air-cavity based FPI sample.

\section{Conclusions}

An in-line micro air-cavity based FPI was demonstrated which was fabricated by multi-step fusion splicing an MPCF to a standard SMF. The air-based FPI was embedded at the splicing point, and it had high-reflectance internal mirrors. So such an FPI exhibited a true and stable sinusoidal interference fringe with relatively high fringe visibility of up to $20 \mathrm{~dB}$. Experimental results showed that such a device could be used as a highly sensitive strain sensor with the sensitivity of 4.5 $\mathrm{pm} / \mu \varepsilon$. Moreover, the temperature compensation may not be required for most of strain sensing applications due to the low thermal coefficient of air trapped in the cavity. Therefore, this kind of FPI will find wide applications due to its advantages of the extremely compact structure, easy fabrication, insensitivity to temperatures, low cost, and high accuracy.

\section{Acknowledgment}

This work is supported by the Nature Science Foundation Project of CQ CSTC under Grant No.cstc2012jjA4007. Assistances and good suggestions of Associate Prof. M. Deng in Chongqing University are appreciated.

Open Access This article is distributed under the terms of the Creative Commons Attribution License which permits any use, distribution, and reproduction in any medium, provided the original author(s) and source are credited.

\section{References}

[1] J. Sirkis, T. A. Berkoff, R. T. Jones, H. Singh, A. D. Kersey, E. J. Friebele, et al., "In-line fiber etalon (ILFE) fiber-optic strain sensors," Journal of Lightwave Technology, 1995, 13(7): 1256-1263.

[2] T. Zhu, D. Wu, M. Liu, and D. Duan, "In-line fiber optic interferometricsensors in single-mode fibers," Sensors, 2012, 12(8): 10430-10449.

[3] X. Wang, J. Xu, Y. Zhu, K. L. Cooper, and A. Wang, "All-fused-silica miniature optical fiber tip pressure sensor," Optics Letters, 2006, 31(7): 885-887.

[4] E. Cibula and D. Donlagic, "In-line short cavity Fabry-Perot strain sensor for quasi distributed measurement utilizing standard OTDR," Optics Express, 2007, 15(14): 8719-8730.

[5] Z. Ran, Y. Rao, H. Deng, and X. Liao, "Miniature in-line photonic crystal fiber etalon fabricated by $157 \mathrm{~nm}$ laser micromachining," Optics Letters, 2007, 32(21): 3071-3073.

[6] Y. Rao, M. Deng, D. Duan, X. Yang, T. Zhu, and G. Cheng, "Micro Fabry-Perot interferometers in silica 
fibers machined by femtosecond laser," Optics Express, 2007, 5(21): 14123-14128.

[7] D. Jauregui-Vazquez, J. M. Estudillo-Ayala, R. Rojas-Laguna, E. Vargas-Rodriguez, J. M. Sierra-Hernandez, J. C. Hernandez-Garcia, et al., "An all fiber intrinsic Fabry-Perot interferometer based on an air-microcavity," Sensors, 2013, 13(5): 6355-6364.

[8] Y. Rao, T. Zhu, X. Yang, and D. Duan, "In-line fiber-optic etalon formed by hollow-core photonic crystal fiber," Optics Letters, 2007, 32(18): 2662-2664.

[9] M. Deng, C. Tang, T. Zhu, and Y. Rao, "PCF-based Fabry-Perot interferometric sensors for strain measurement at high temperatures," IEEE Photonics Technology Letters, 2011, 23(11): 700-703.

[10] E. Li, G. Peng, and X. Ding, "High spatial resolution fiber-optic Fizeau interferometric strain sensor based on all-fiber spherical microcavity," Applied Physics Letters, 2008, 92: 101117-1-101117-3.

[11] P. F. C. Antunes, M. F. F. Domingues, N. J. Alberto, and P. S. Andre, "Optical fiber micro-cavity strain sensors produced by the catastrophic fuse effect," IEEE Photonics Technology Letters, 2014, 26(1): 78-81.

[12] F. C. Favero, L. Araujo, G. Bouwmans, V. Finazzi, J. Villatoro, and V. Pruneri, "Spheroidal Fabry-Perot microcavities in optical fibers for high-sensitivity sensing," Optics Express, 2012, 20(7): 7112-7118.

[13] L. Xiao, M. S. Demokan, W. Jin, Y. Wang, and C. Zhao, "Fusion splicing photonic crystal fibers and conventional single-mode fibers: microhole collapse effect," Journal of Lightwave Technology, 2007, 25(11): 3563-3574. 